\title{
openheart Reducing patient delay with symptoms of acute coronary syndrome: a research protocol for a systematic review of previous interventions to investigate which behaviour change techniques are associated with effective interventions
}

\author{
Barbara Farquharson, ${ }^{1}$ Stephan Dombrowski, ${ }^{2}$ Alex Pollock, ${ }^{3}$ Marie Johnston, ${ }^{4}$ \\ Shaun Treweek, ${ }^{5}$ Brian Williams, ${ }^{1}$ Karen Smith, ${ }^{6}$ Nadine Dougall, ${ }^{1}$ Claire Jones, ${ }^{7}$ \\ Stuart Pringle ${ }^{8}$
}

To cite: Farquharson $B$, Dombrowski S, Pollock A, et al. Reducing patient delay with symptoms of acute coronary syndrome: a research protocol for a systematic review of previous interventions to investigate which behaviour change techniques are associated with effective interventions. Open Heart 2014;1:e00079. doi:10.1136/openhrt-2014000079

Received 21 February 2014 Revised 13 June 2014 Accepted 15 July 2014

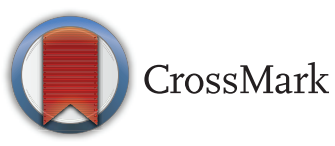

For numbered affiliations see end of article.

Correspondence to Dr Barbara Farquharson; barbara.farquharson1@stir. ac.uk

\section{ABSTRACT}

Introduction: Delay to presentation with symptoms of acute coronary syndrome (ACS) is common meaning many fail to achieve optimal benefit from treatments. Interventions have had variable success in reducing delay. Evidence suggests inclusion of behaviour change techniques (BCTs) may improve effectiveness of interventions but this has not yet been systematically evaluated. Data from other time-critical conditions may be relevant.

Methods and analysis: A systematic review will be undertaken to identify which BCTs are associated with effective interventions to reduce patient delay (or prompt rapid help-seeking) among people with time-critical conditions (eg, chest pain, ACS, lumps, stroke, cancer and meningitis). A systematic search of a wide range of databases (including Cochrane Library, MEDLINE, EMBASE, CINAHL, Psyclnfo) and grey literature will be undertaken to identify all relevant intervention studies (randomised controlled trials, controlled clinical trials and cohort studies). Two independent reviewers will screen abstracts to identify relevant studies, apply inclusion criteria to full papers, assess methodological quality and extract data

Primary outcome measure: Change in patient decision time BCTs reported in each of the included studies will be categorised and presented according to the latest reliable taxonomy. Results of included studies will be synthesised, exploring relationships between inclusion of each BCT and effectiveness of the overall intervention. Where possible, means and SDs for differences in delay time will be calculated and combined within meta-analyses to derive a standardised mean difference and $95 \% \mathrm{Cl}$. Analysis of (1) all time-critical and (2) ACS-only interventions will be undertaken.

Ethics and dissemination: No ethical issues are anticipated. Results will be submitted for publication in a relevant peer-reviewed journal.

\section{KEY MESSAGES}

What is already known about this subject?

- Interventions to reduce patient delay in acute coronary syndrome have had variable success. Inclusion of behaviour change techniques is likely to improve the effectiveness of interventions.

What does this study add?

- This study will systematically identify the behaviour change techniques which have been used in previous successful interventions to reduce patient delay in time-critical conditions and subsequently inform the content of future interventions.

How might this impact on clinical practice?

- An intervention that successfully reduces patient delay will help ensure that more patients benefit optimally from available but time-dependent treatments.

\section{INTRODUCTION}

Time to treatment in acute coronary syndrome (ACS) has been recognised as critical for many years. ${ }^{1}$ Despite this, there is widespread evidence that delay to receipt of treatment is common. $^{2-6}$ Reported average time-totreatment ranges from 1.5 to $6 \mathrm{~h}^{6} 7$ and as many as a third of patients arrive too late to be eligible for optimal treatment. ${ }^{8}$ Research shows that the interval which contributes most to prehospital time is patient decision time (frequently referred to as 'patient delay') that is, the interval between onset of symptoms and seeking medical help. ${ }^{2}$ Reductions in patient delay have the potential to lead to significant reductions in mortality for ACS. Indeed, recent 
guidelines of the European Society of Cardiology ${ }^{9}$ identified the prehospital phase as the most critical in reducing mortality and reiterated that efforts must be made to reduce patient delay. In particular, people with existing coronary heart disease (CHD) are a population most at risk of experiencing an episode of $\mathrm{ACS}^{10}$ and therefore a very important group in which to ensure prompt help-seeking. Furthermore, the recurrence of symptoms following an acute event (eg, post-MI angina) indicates high risk and is likely to prompt additional clinical investigation and treatment. ${ }^{10}$ Thus while it is desirable to reduce delay in the general population, it is particularly vital to effectively reduce delay in those with existing CHD.

Despite the recognised need to reduce patient delay among people with CHD it is not yet clear how best this might be achieved. ${ }^{11}$ Previous interventions to reduce patient delay in ACS, some large and comprehensive, have been largely unsuccessful in changing behaviour. ${ }^{12-14}$ There is a growing body of evidence that suggests incorporating relevant psychological theory, ${ }^{15-18}$ and embedding evidence-based behaviour change techniques $(\mathrm{BCTs})^{19}$ (eg, goal-setting, action planning) in interventions is more likely to lead to successful behaviour change. An intervention based on psychological theory and utilising established BCTs has been effective in reducing patient delay with symptoms of lung cancer, another time-sensitive condition. ${ }^{20}{ }^{21}$ However, to date, interventions aimed at reducing delay in people with ACS have tended not to apply relevant psychological theory $^{22}$ have not systematically included specific BCTs ${ }^{23}$ or fully considered optimum modes of delivery. To ensure potentially valuable relevant evidence relating to other conditions is not excluded, the systematic review will include studies of all interventions to reduce patient delay (or prompt rapid help-seeking) among people with symptoms or conditions where time to treatment is critical (eg, chest pain, lumps, stroke, cancer, ACS and meningitis). However, in order to also obtain an ACS perspective, analysis will be performed for all eligible papers and then repeated for only ACS papers.

This protocol describes a systematic review which will be undertaken by the authors as part of a study to develop an intervention to reduce delay in ACS (peerreviewed and funded by the Health Services and Population Health research committee of the Chief Scientist Office, Scottish Government).

The aim of the systematic review is to: identify which BCTs are associated with effective interventions to reduce patient delay (or prompt rapid help-seeking) among people with symptoms or conditions where time to treatment is critical.

\section{METHODS AND ANALYSIS \\ Study inclusion criteria}

Types of study

Any intervention study (including randomised controlled trials, controlled clinical trials and cohort studies) which include a measure of patient delay/time to presentation (ie, behaviour).

\section{Types of participants}

Adults ( $>18$ years) with symptoms or conditions where time to treatment is critical (ie, timing of presentation has a demonstrated effect on mortality).

\section{Types of interventions}

Any intervention where the stated aim was to reduce patient delay/time to presentation to health services (eg, patient education intervention or multi-media public health campaign).

\section{Types of outcome measure}

Primary outcome of interest is patient delay time (ie, interval between the onset of symptoms and seeking medical help). Studies must include a measure of patient delay time to be included. The following secondary outcomes: intentions to seek help without delay; number and type of presentations with symptoms (general practitioner (GP), hospital, ambulance); number of deaths and adverse events will also be examined.

\section{Study exclusion criteria}

Non-English language papers will not be excluded, and efforts will be made to obtain timely translations. However, we anticipate it may not always be possible to obtain translations within the time constraints of the project.

\section{Search strategy}

We will carry out a systematic search of electronic databases, using a sensitive search strategy developed in collaboration with expert medical librarians. This will include the following MESH terms: "chest pain", "myocardial infarction", "acute coronary syndrome", "stroke", "neoplasms", "meningitis", "dyspnea", "signs and symptoms", "intervention studies" and the keywords "time to presentation", "delay", "patient delay", and "pre-hospital". Electronic databases will include the Cochrane Library, MEDLINE, EMBASE, Cumulative Index to Nursing and Allied Health Literature, PsycInfo, and National Research Register. We will screen reference lists of all relevant articles and use citation index databases (Science Citation Index (SCI), Social Sciences Citation Index (SSCI) and Arts and Humanities Citation Index (A\&HCI) for citation tracking of relevant included studies. We will not limit the search based on language of publication or year of publication. We will also endeavour to identify any relevant 'grey literature' by emailing researchers working in this field to try to identify any unpublished, on-going or recently completed research.

\section{Study selection}

One reviewer will initially consider the titles of the studies identified in the search and exclude any which are 'obviously irrelevant'. Two independent reviewers will then screen all remaining abstracts identified in the searching. 
Full papers of any studies considered to be potentially relevant by either of the reviewers will be obtained. Two independent reviewers will apply the inclusion criteria to these full papers. Where there are disagreements consensus will be reached through discussion, using a third reviewer where necessary. We will also contact authors of studies where there is insufficient information to assess whether the study should be included. Frequency of disagreement and reasons for excluding studies considered at the full paper stage will be documented.

\section{Assessment of methodological quality}

Methodological quality of studies will be assessed by two independent reviewers and documented using assessment tools relevant to the type of study (ie, Cochrane risk of bias tool ${ }^{24}$ and CASP tools ${ }^{25}$ ). Specifically, selection bias, performance bias, attrition bias and detection bias will be assessed.

\section{Data extraction}

Data extraction will be guided by the CONSORT ${ }^{26}$ and $\mathrm{TIDieR}^{27}$ guidelines and an assessment of the completeness of reporting undertaken. The following data will be extracted from each included study (study design; study recruitment, participant population; number of participants; comparison interventions;; study location country; setting; mode of delivery (including contact frequency, duration and intensity; materials used; details of intervener(s)); BCTs included in intervention and control/ comparison conditions (using BCTTv1 taxonomy, ${ }^{28}$ see below); intervention fidelity assessment and results; outcomes assessed (what (ie, change in median (IQR) patient decision time (or mean (SD), CI, p values as available) and when).

BCTs reported in each of the included studies will be categorised by two independent reviewers who have undergone training in the reliable identification of BCTs. BCTs will be presented according to the most comprehensive and reliable taxonomy ${ }^{28}$ of BCTs in behavioural interventions and will be based on the most comprehensive published intervention descriptions. All inter-rater agreements for risk of bias items will be assessed using $\kappa$.

\section{Primary outcome measure}

Patient decision time.

\section{Secondary outcome measures}

Intention to seek help without delay scores (units/ scale), number of presentations with symptoms of ACS (GP, hospital, ambulance and timeframe of same).

\section{Synthesis and analysis}

Data from included studies will be analysed narratively and meta-analyses with subgroup analyses will be conducted where the data is appropriate for such analysis. All meta-analyses will be conducted using random-effects models. Where possible, means and SDs for differences in delay time (between intervention and control or before and after) will be calculated for all trials and combined within meta-analyses to derive a standardised mean difference and 95\% CI to express the difference between intervention and control in terms of SD units. We will request means and SDs from authors of studies which do not report means and SDs, but if this is not possible we will convert medians and IQR (if reported) to approximate means and SDs using a standardised conversion. ${ }^{29}$ Skewness for each study will be estimated and tabulated using the criteria suggested by Altman and Bland. ${ }^{30}$ Where data are severely skewed, (eg, Kolmogorov-Smirnov test $\mathrm{p} \leq 0.01$ ), the study will be not included in the meta-analysis. Degree of inconsistency will be assessed using $\mathrm{I}^{2} .{ }^{31}$ Moderate levels of heterogeneity $\left(\mathrm{I}^{2}>30 \%\right)$ within main effects analyses will be followed up using subgroup analyses to examine between-study variability. The following planned subgroup analyses will be performed:

- BCT-based subgroup analysis examining associations between the presence of a technique, or cluster of techniques, and effectiveness.

- Mode of delivery subgroup analyses examining associations between the presence of delivery features and effectiveness.

- Risk of bias based subgroup analyses examining whether allocation concealment and blinding of outcome assessment are associated with effectiveness.

Univariate metaregressions will be performed to assess the effects of predictor variables on delay time. Funnel plots will be inspected for symmetry and sensitivity analyses conducted removing any outliers. The characteristics and results of individual studies not included in the meta-analyses will be tabulated, including all data extracted and the quality assessment.

Author affiliations

${ }^{1}$ Nursing, Midwifery and Allied Health Professionals Research Unit, University of Stirling, Stirling, UK

${ }^{2}$ School of Psychology, University of Stirling, Stirling, UK

${ }^{3}$ Nursing, Midwifery and Allied Health Professionals Research Unit, Glasgow Caledonian University, Glasgow, UK

${ }^{4}$ Institute of Applied Health Sciences, University of Aberdeen, Aberdeen, UK

${ }^{5}$ Health Services Research Unit, University of Aberdeen, Aberdeen, UK

${ }^{6}$ School of Nursing and Midwifery, University of Dundee, Dundee, UK

${ }^{7}$ Health Informatics Centre, University of Dundee, Dundee, UK

${ }^{8}$ Cardiology Department, NHS Tayside, Dundee, UK

Contributors The methods for the systematic review were originally written by $\mathrm{BF}$ and $\mathrm{AP}$ and revised following review by MJ, BW, KS, SD, ST, CJ, ND and SP.

Funding This work was supported by the Chief Scientist Office, Scotland: grant number $\mathrm{CZH} / 4 / 1025$.

Competing interests None.

Provenance and peer review Not commissioned; externally peer reviewed.

Data sharing statement No additional data are available.

Open Access This is an Open Access article distributed in accordance with the Creative Commons Attribution Non Commercial (CC BY-NC 3.0) license, which permits others to distribute, remix, adapt, build upon this work noncommercially, and license their derivative works on different terms, provided the original work is properly cited and the use is non-commercial. See: http:// creativecommons.org/licenses/by-nc/3.0/ 


\section{REFERENCES}

1. Goldberg R, Yarzebski J, Lessard D, et al. Decade-long trends and factors associated with time to hospital presentation in patients with acute myocardial infarction: the Worcester Heart Attack Study. Arch Intern Med 2000;160:3217-23.

2. Gruppo Italiano per lo Studio della Sopravvivenza nell'Infarto (GISSI). Epidemiology of avoidable delay in the care of patients with acute myocardial infarction in Italy: a GISSI-Generated Study. Arch Intern Med 1995;155:1481-8.

3. Gibler BW, Armstrong PW, Ohman EM, et al. Persistance of delays in presentation and treatment for patients with acute myocardial infarction: the GUSTO-I and GUSTO-III experience. Ann Emerg Med 2002;39:123-30.

4. Saczynski JS, Yarzebski J, Lessard D, et al. Trends in prehospital delay in patients with acute myocardial infarction (from the Worcester Heart Attack Study). Am J Cardiol 2008;102:1589-94.

5. O'Carroll R, Smith K, Grubb N, et al. Psychological factors associated with delay in attending hospital following myocardial infarction. J Psychosom Res 2001;51:611-14.

6. Dracup K, Moser D, McKinley S, et al. An international perspective of the time to presentation with myocardial infarction. J Nurs Scholarsh 2003;35:317-23.

7. O'Donnell S, McKee G, Mooney M, et al. Slow-onset and fast-onset symptom presentations in Acute Coronary Syndrome (ACS): new perspectives on pre-hospital delay in patients with ACS. J Emerg Med 2014;46:507-15.

8. Eagle KA, Goodman SG, Avezum A, et al. Practice variation and missed opportunities for reperfusion in ST-segment-elevation myocardial infarction: findings from the Global Registry of Acute Coronary Events (GRACE). Lancet 2002;359:373-7.

9. Tubaro M, Danchin N, Goldstein P, et al. Pre-hospital treatment of STEMI patients. A scientific statement of the Working Group Acute Cardiac Care of the European Society of Cardiology. Acute Card Care 2011;13:56-67.

10. Anderson JL, Adams CD, Antman EM. ACC/AHA 2007 guidelines for the management of patients with unstable angina/non ST-elevation myocardial infarction: a report of the American College of Cardiology/American Heart Association Task Force on Practice Guidelines (Writing Committee to Revise the 2002 Guidelines for the Management of Patients With Unstable Angina/ Non ST-Elevation Myocardial Infarction). J Am Coll Cardiol 2007;50 e1-e157.

11. Ting $\mathrm{HH}$, Bradley $\mathrm{EH}$. Patient education to reduce prehospital delay time in acute coronary syndrome: necessary but not sufficient. Circ Cardiovasc Qual Outcomes 2009;2:522-3.

12. Dracup K, McKinley S, Riegel B, et al. A randomized clinical trial to reduce patient prehospital delay to treatment in acute coronary syndrome. Circ Cardiovasc Qual Outcomes 2009;2:524-32.

13. Kainth A, Hewitt A, Sowden A, et al. Systematic review of interventions to reduce delay in patients with suspected heart attack Emerg Med J 2004;21:506-8.

14. Luepker RV, Raczynski JM, Osganian S, et al. Effect of a community intervention on patient delay and emergency medica service use in acute coronary heart disease: the Rapid Early Action for Coronary Treatment (REACT) Trial. JAMA 2000;284:60.

15. Michie S, Johnston M, Francis J, et al. From theory to intervention: mapping theoretically derived behavioural determinants to behaviour change techniques. Appl Psychol 2008;57:660-80.

16. Craig P, Dieppe $\mathrm{P}$, Macintyre S, et al. Developing and evaluating complex interventions: the new medical research council guidance. BMJ 2008;337:979-83

17. Campbell M, Fitzpatrick R, Haines A, et al. Framework for design and evaluation of complex interventions to improve health. BMJ 2000;321:694-6.

18. Campbell NC, Murray E, Darbyshire J, et al. Designing and evaluating complex interventions to improve health care. BMJ 2007;334:455-9.

19. Webb TL, Joseph J, Yardley L, et al. Using the internet to promote health behavior change: a systematic review and meta-analysis of the impact of theoretical basis, use of behavior change techniques, and mode of delivery on efficacy. J Med Internet Res 2010;12:e4.

20. Smith S, Fielding S, Murchie P, et al. Reducing time before consulting with symptoms of lung cancer: randomised controlled trial. Br J Gen Pract 2013;63:e47-54.

21. Smith S, Murchie P, Devereux G, et al. Developing a complex intervention to reduce time to presentation with symptoms of lung cancer. Br J Gen Pract 2012;62:e605-15.

22. Khraim FM, Carey MG. Review: Predictors of pre-hospital delay among patients with acute myocardial infarction. Patient Educ Couns 2009;75:155-61.

23. Abraham $\mathrm{C}$, Michie $\mathrm{S}$. A taxonomy of behaviour change techniques used in interventions. Health Psychol 2008;27:379-87.

24. Higgins JPT, Altman DG, eds. Chapter 8: assessing risk of bias in included studies. In: Higgins JPT, Green S, eds. Cochrane Handbook for Systematic Reviews of Interventions Version 5.0.1 [updated September 2008]. The Cochrane Collaboration, 2008. http://www.cochrane-handbook.org

25. Critical Appraisal Skills Programme. http://www.casp-uk.net/\#! casp-tools-checklists/c18f8 (accessed 28 Feb 2014).

26. Schulz KF, Altman DG, Moher D; for the CONSORT Group. CONSORT 2010 Statement: updated guidelines for reporting paralle group randomised trials. BMJ 2010;340:c332.

27. Hoffmann, Glasziou PP, Boutron I, et al. Better reporting of interventions: the Template for Intervention Description and Replication (TIDieR) checklist and guide'. BMJ 2014;348:g1687.

28. Michie S, Richardson $M$, Johnston $M$, et al. The behavior change technique taxonomy (v1) of 93 hierarchically clustered techniques: building an international consensus for the reporting of behavior change interventions. Ann Behav Med 2013;46:81-95.

29. Hozo S, Djbegovic B, Hozo I. Estimating the mean and variance from the median, range, and the size of the sample. BMC Med Res Methodol 2005;5:13.

30. Altman DB, Bland JB. Detecting skewness from summary information. BMJ 1996;313:1200.

31. Higgins JPT, Thompson SG, Deeks JJ, et al. Measuring inconsistency in meta-analyses. BMJ 2003;327:557-60. 